Series A

\author{
I. MATHEMATICA
}

442

\title{
ON A CLASS OF KLEINIAN GROUPS
}

BY

BERNARD MASKIT

H ELSINKI 1969

S UOMALAINEN TIEDEAKATEM A

doi:10.5186/aasfm.1969.442 
Communicated 13 December 1968 by L. V. Ahlfors and P. J. Myrberg 


\section{ON A CLASS OF KLEINIAN GROUPS}

Let $\hat{C}$ denote the Riemann sphere $(\hat{C}=C \cup\{\infty\})$. A Kleinian group $G$ is a group of Mobius transformations (directly conformal self maps of $\hat{C})$ which is discontinuous somewhere. The set of points at which $G$ is discontinuous is called the regular set and is denoted by $R(G)$. A connected component of $R(G)$ is called a component of $G$.

Every Mobius transformation $g$ can be written in the form $z \rightarrow$ $(a z+b) /(c z+d), a d-b c=1 . g$ determines $a, b, c$, and $d$ up to sign, and $\operatorname{tr}^{2}(g)=(a+d)^{2}$ is well defined. $g$ is called loxodromic if $\operatorname{tr}^{2}(g)$ does not lie in the closed real segment $[-4,4]$.

A Kleinian group $G$ is called a $\pi^{p}$ group if every $g \in G, g \neq 1$, is loxodromic, and $G$ is isomorphic to the fundamental group of a closed orientable surface of genus $p, p \geq 2$. $G$ then has a presentation of the form $a_{1}, b_{1}, \ldots, a_{p}, b_{p}: \pi_{i=1}^{p}\left[a_{i}, b_{i}\right]$.

The main result of this paper (theorem 3 ) is that every $\pi^{p}$-group has a simply connected invariant component.

Let $\Gamma$ be a Fuchsian $\pi^{P}$-group acting on the lower half plane $L$. A quadratic differential on $\Gamma$ is a holomorphic function $\varphi$ on $L$, where $\varphi(\gamma(z))\left(\gamma^{\prime}(z)\right)^{2}=\varphi(z)$, for all $\gamma \in \Gamma$. The space of all quadratic differentials on $\Gamma$, with norm

$$
\|\varphi\|=\sup _{z=x+i y \in L}\left|y^{2} \varphi(z)\right|
$$

is denoted by $B(\Gamma)$.

Every $\varphi \in B(\Gamma)$ induces a homomorphism $x_{\varphi}$ of $I$ into the group of all Mobius transformations as follows. Given $\varphi$, there is a unique meromorphic function $w_{\text {rs }}$ on $L$, where the Schwartzian derivative of $w_{q}$ equals $\varphi$, and near $-i, w_{q f}(z)=1 /(z+i)+0(i z+i)$. For each $\gamma \in \Gamma$, there is a unique Mobius transformation $x_{q}(\gamma)$ so that

$$
w_{\varphi} \circ \gamma(z)=x_{\varphi}(\gamma) \circ w_{q}(z), \text { for all } z \in L .
$$

A point $\varphi \in B(T)$ is called regular if $x_{i}$ is an isomorphism, and if every non-trivial element of $x_{r}(\Gamma)$ is loxodromic. It was observed by Kra [5], that almost all $\varphi \in B(\Gamma)$ are regular. 
Our main result asserts that if $\varphi \in B(\Gamma)$ is regular and $x_{\varphi}(\Gamma)$ is discontinuous, then $x_{r}(T)$ has a simply connected invariant component. One might suppose that this would imply that $w_{p}$ is univalent in $L$. Theorem 5 shows that this is not necessarily true.

Throughout this paper we will use various well known facts about quadratic differentials, Fuchsian and quasi-Fuchsian groups, and quasiconformal mappings. The reader is referred to [2] for the basic definitions and proofs.

The author would like to thank Irwin Kra for several conversations during which he raised these questions.

Theorem 1: Let $G$ be a Kleinian group isomorphic to the fundamental group of a closed orientable surface of genus $p \geq 2$. Suppose that $G$ has an invariant component $R_{0}$. Then $R_{0}$ is simply connected and $R_{0} / G$ is a closed orientable surface of genus $p$.

Proof: $G$ is finitely generated. By Ahlfors theorem [1] $S=R_{0} / G$ is a closed Riemann surface from which a finite number of points have been removed.

Assume that $R_{0}$ is not simply connected. Then by the planarity theorem [6], there is a set $w_{1}, \ldots, w_{q}, q \geq 1$, of simple disjoint loops on $S$ with the following properties. No $w_{i}$ bounds either a disc or a punctured disc on $S$. Each $w_{i}$ lifts to a loop on $R_{0} . R_{0}$ with group $G$ is the highest regular covering of $S$ for which $w_{1}, \ldots, w_{q}$ lift to loops.

Let $K^{\prime}$ be the 2 -complex obtained from $S$ by passing abstract dises $p_{1}, \ldots, p_{q}$ through $w_{1}, \ldots, w_{q}$. By lifting $p_{1}, \ldots, p_{q}$, to abstract discs sewed onto $R_{0}$, in all possible ways, we see that $G$ is isomorphic to $\pi_{1}\left(K^{\prime}\right)$.

There are elementary homotopy equivalences to show that if $q \geq 1$, then $\pi_{1}\left(K^{\prime}\right)$ is a non-trivial free product (see [6] pgs. $352-3$ and [7] pg. 228). Briefly, if $w_{i}$ is dividing, contract $p_{i}$ to a point; if $w_{i}$ is nondividing, contract $p_{i}$ to a point, pull the two pieces of surface apart stretching the point into a 1-cell, and then pull the endpoints of the 1-cell together. The resulting 2-complex $K$ is clearly a wedge product of homotopically non-trivial components. Hence $\tau_{1}\left(K^{\prime}\right)=\tau_{1}(K)$ is a nontrivial free product.

$G$ is isomorphic to the fundamental group of a closed orientable surface, hence every subgroup of $G$ is either free or of finite index. If $G$ were a non-trivial free product, $G=A * B$, then $A$ and $B$, being infinite groups, would both have infinite index in $G$. Hence $A$ and $B$ would both be free, and so $G$ would be free, which it isn't.

We conclude that $R_{0}$ is simply connected. It follows at once from the classification of surfaces that $S$ is a closed Riemann surface of genus $p$.

The following theorem is a special case of theorem 4 in [8]. 
Theorem 2: Let $G$ be a $\pi^{p}$-group with a simply connected invariant component $R_{0}$. Then either

1) $G$ is degenerate; i.e. $R(G)=R_{0}$, or

2) $G$ is quasi-Fuchsian; i.e. there is a Fuchsian group $\Gamma$, and a quasicon-

formal homeomorphism $w: \hat{C} \rightarrow \hat{C}$ so that $G=w \circ \Gamma \circ w^{-1}$.

Theorem 3: Let $G$ be a $\pi^{p}$-group. Then $G$ has a simply connected invariant component.

Proof: Assume not. Then $G$ has at least two components $R_{0}$ and $R_{1}$. Let $H$ be the subgroup of $G$ keeping $R_{\jmath}$ invariant.

$R_{0} / H$ is a connected component of $R(G) / G$. By Ahlfors theorem [1], $R_{0} / H$ is a finite surface, and so $H$ is finitely generated.

$H$ is a subgroup of $G$, and so $H$ is either free, or $H$ is a $\pi^{q}$-group for some $q>p$. Is $H$ were free, then by [7], $H$ would be a Schottky group, contradicting the fact that $H$ has at least two components.

$H$ has an invariant component $R_{0}$. By theorem $1, R_{0}$ is simply connected. By assumption $R_{0}$ is a proper subset of $R(G) \subset R(H)$ and so, by theorem 2, $H$ is quasi-Fuchsian. There is then a Fuchsian group $\Lambda^{*}$, acting on the upper half plane $U$, and a global quasiconformal homeomorphism $W$, where $W(U)=R_{0}$, and $W \circ \Lambda \circ W^{-1}=H$. Let $R_{1}^{\prime}=W(L)$.

$H$ is of finite index in $G$, hence $R(G)=R(H)=R_{0}+R_{1}^{\prime} . R_{0}$ and $R_{1}^{\prime}$ are both invariant under $H$, hence $[G: H]=2$, and, using the Riemann-Hurwitz formula, $q=2 p-1$.

Let $g$ be some element of $G-H$. Then $g \circ h \circ g^{-1} \in H$. Let $r$ denote reflection in the real axis. Then $W \circ r \circ W^{-1}$ commutes with every element of $H$. It follows that $\bar{g}=g \circ W \circ r \circ W^{-1}$ is an orientation reversing homeomorphism of $R_{0}$, where $\bar{g} \circ h \circ \bar{g}^{-1} \in H$ for every $h \in H$.

Let $\Gamma$ be some Fuchsian $\pi^{p}$-group acting on $U$. Let $\psi: G \rightarrow \Gamma$ be some isomorphism, and let $\Delta=\psi(H)$. $U / \Delta$ and $S=R_{0} / H$ are both closed Riemann surfaces of genus $q$, hence they are homeomorphic. Every isomorphism of $\pi_{1}(S)$ is induced by a homeomorphism of $S$ (Nielsen [9], see also Zieschang [10]). Hence there is a homeomorphism $V: U \rightarrow R_{0}$ so that $V^{-1} \circ h \circ V=\psi(h)$ for every $h \in H$.

Let $\gamma=\psi(g)$, and set $\gamma^{*}=\gamma^{-1} \circ V^{-1} \circ \bar{g} \circ \dot{V} . \gamma^{*}$ is an orientation reversing homeomorphism of $U$, and $\gamma^{*}$ commutes with every element of $\Delta$, which is absurd.

Theorem 4: There exists a Kleinian group $G$, which is isomorphic to the fundamental group of a closed orientable surface, and which has no invariant component.

Proof: We write the Mobius transformation $z \rightarrow(\alpha z+\beta) /(\gamma z+\delta)$ as $(\alpha, \beta ; \gamma, \delta)$. Let $a=(1,-4 ; 0,1), b=(1,0 ; 1,1), c=(-3,4 ;-1,1)$, 
$d=(-i, 2 i ;-1,2+i), e=(1+4 i, 16 ; 1,1-4 i), f=(3+4 i, 12-16 i ;$ $1,-1-4 i)$, and $g=(3 i, 8-6 i ; 1,-2-3 i)$. Let $G$ be the group generated by $a, \ldots, g$. We remark that $G$ is a subgroup of the Picard group.

Let $C_{1}$ and $C_{2}$ be the lines $R e z=3$ and $R e z=-1$, respectively. Let $C_{3}, \ldots, C_{12}$ be the circles of radius 1 with centers at $-1,1,3$, $2+i, i,-1+4 i, 1+4 i, 3+4 i, 2+3 i, 3 i$ respectively. Let $P_{1} \ldots, P_{12}$ be the non-Euclidean planes in the upper half space, whose boundaries are the circles $C_{1}, \ldots, C_{12}$, respectively. Observe that $a\left(P_{1}\right)=P_{2}, b\left(P_{3}\right)=P_{4}, c\left(P_{4}\right)=P_{5}, d\left(P_{6}\right)=P_{7}, e\left(P_{8}\right)=P_{9}, f\left(P_{9}\right)=P_{10}$, and $g\left(P_{11}\right)=P_{12}$. Splitting $P_{4}$ and $P_{9}$ into two pieces each, using the plane bounded by $\operatorname{Re} z=1$, we get a polyhedron $Q$ whose sides are pairwise identified by $a, \ldots, g$. We also observe that any two interesting sides of $Q$ meet an angle of $\pi / 2$ or $\pi$.

By Poincaré's theorem (see [3] pg. 174-8), $Q$ is a fundamental polyhedron for $G$. It follows that $R(G) / G$ is the disjoint union of four 3-times punctured spheres. It also follows that $c b a=d^{-1} b d c=a f e=g^{-1}$ egf $=1$ is a complete set of relations for $G$. One sees at once that $G$ is generated by $b, d, e$, and $g$, and that these generators satisfy the one defining relation $b^{-1} d^{-1} b d g^{-1} e^{-1} g e=1$.

Remark 1: $G$ constructed above is isomorphic to a $\pi^{2}$-group. For every $p=3,4, \ldots$, there is a Kleinian group which is isomorphic to a $\pi^{p}$-group, and which has no invariant component. The construction of such groups is considerably more complicated, and not worth the effort.

Theorem 5: Let $G$ be a Fuchsian $\pi^{p}$-group. Then there is a Mobius transformation a, a Fuchsian $\pi^{p}$-group $\Gamma$, and a regular $\varphi \in B(\Gamma)$, so that $X_{\text {rr }}(\Gamma)=a \circ G \circ a^{-1}$, and so that $w_{\varphi}$ is not univalent in $L$.

Proof: We normalize $G$ so that the negative imaginary axis projects onto a simple closed curve on $L / G$. There is then a smallest $\varrho>1$ so that $z \rightarrow \varrho z$ is an element of $G$. There is also an $x>0$ so that no two points of

$$
B=\{z|1 \leq| z \mid<\varrho, 3 \pi / 2-\alpha \leq \arg z \leq 3 \pi / 2+x\}
$$

are equivalent under $G$.

A fundamental set for $G$ acting on $L$ is a set $D \subset L$ so that the natural projection $p: L \rightarrow L / G$, when restricted to $D$, is a one-to-one map of $D$ onto $L / G$. It is well known that there is such a fundamental set $D$ with the following additional properties. $D$ is bounded by a nonEuclidean polygon whose sides are pairwise identified by elements of $G$; these elements generate $G . B \subset D \subset\{z|1 \leq| z \mid<\varrho\}$.

Let $v(\Theta)$ be a $C^{\infty}$ real valued function with the following properties. $v$ is a diffeomorphism of $[3 \pi / 2-\alpha, 3 \pi / 2+\alpha]$ onto $[3 \pi / 2-\alpha, 7 \pi / 2+\alpha)$. 
$v(3 \pi / 2-\alpha)=3 \pi / 2-\alpha v^{\prime}(3 \pi / 2-\alpha)=v^{\prime}(3 \pi / 2+\alpha)=1$. All the higher derivatives of $v$ vanish at the endpoints.

We now define a function $f$ on $L$. For $z \in D-B$, set $f(z)=z$. For $z=r e^{i \Theta} \in B$, set $f(z)=r e^{i v(\theta)}$. If $z \in L-D$, then there is a $g \in G$, and a $w \in D$, so that $g(w)=z$; set $f \circ g(w)=g \circ f(w)$. One sees at once that $f$ is a $C^{\infty}$ map of $L$ onto $\hat{C}, f$ is a local homeomorphism, and for every $g \in G, f \circ g=g \circ f$.

For $z \in L$, set $\mu(z)=f_{\tilde{z}} / f_{z}$. Observe that for $g \in G$,

$$
\mu(g(z)) g^{\prime}(z) / \overline{g^{\prime}(z)}=\mu(z) .
$$

By the compactness of $D$, there is a $k<1$, so that $|\mu(z)| \leq k$. For $z \in U$, set $\mu(z)=\overline{\mu(z)}$. For $z \in R \cup\{\infty\}$, set $\mu(z)=0$.

Let $w$ be the global quasiconformal homeomorphism satisfying $w_{\bar{z}}=\mu(z) w_{z}, w(O)=O, w(1)=1, w(\infty)=\infty$. Then $w(L)=L$, and for every $g \in G, w \circ g \circ w^{-1}$ is a Möbius transformation. Set $\Gamma=w \circ G \circ w^{-1}$. $f \circ w^{-1}$ is meromorphic in $L$, and is a local homeomorphism; hence $\varphi$, the Schwartzian derivative of $f \circ w^{-1}$ is holomorphic. $\varphi \in B(\Gamma)$, since $\left(f \circ w^{-1}\right) \circ\left(w \circ g \circ w^{-1}\right)=g \circ\left(f \circ w^{-1}\right) . \quad w_{p}$ and $f \circ w^{-1}$ have the same Schwartzian derivative and so there is a Möbius transformation $a$ so that $w_{\varphi}=a \circ\left(f \circ w^{-1}\right)$. It follows that $x_{q}(\Gamma)=a \circ G \circ a^{-1}$.

Remark 2: There is a simple modification of the above construction to yield the same result starting with a quasi-Fuchsian group $G$. There is also an obvious modification of the construction to yield a sequence of quadratic differentials $\varphi_{n}$ in different spaces $B\left(\Gamma_{n}\right)$, with the same properties.

Remark 3: Let $\varphi \in B(\Gamma)$ be as in theorem 5. Then by quasiconformal stability [4], there is a neighborhood $U$ of $\varphi$, so that for every $\psi \in U$, $x_{\psi}(\Gamma)$ is quasi-Fuchsian and $w_{\psi}$ is not univalent.

Remark 4: Let $\Gamma$ be a Fuchsian $\pi^{p}$-group. It is not known whether or not there is a regular $\varphi \in B(\Gamma)$ so that $x_{\varphi}$ is not univalent.

Remark 5: Let $\varphi \in B(\Gamma)$ be as in theorem 5. It was shown by Kra [5] that $w_{\varphi}(L)=\hat{C}$, and that $w_{\varphi}$ is not a cover map.

Massachusetts Institute of Technology

Cambridge, Mass. U.S.A. 


\section{References}

[1] Ahlfors, L. V.: Finitely generated Kleinian groups, - Am. J. of Math. 86 (1964) 413-429.

[2] - -- Lectures on quasiconformal mappings. - van Nostrand, Princeton, N. J., 1966.

[3] Appell, P. et Goursat, E.: Théorie des fonctions algébriques et des transcendantes qui s'y rattachent II, par P. Fatou. - Gauthier-Villars, Paris, 1930.

[4] Bers, L.: On boundaries of Teichmuller spaces and on Kleinian groups I, (to appear).

[5] Kra, I.: Deformations of Fuchsian groups. - Duke Math. J. (to appear).

[6] Maskit, B.: A theorem on planar coverings of surfaces with applications to 3 manifolds. - Annals of Math. 81 (1965), 341-355.

[7] -"- A characterization of Schottky groups. - J. d'Anal. Math. 19 (1967), $227-230$.

[8] - - - On boundaries of Teichmuller spaces and on Kleinian groups II, (to appear).

[9] Nielsen, J.: Untersuchungen zur Topologie der geschlossenen zweiseitigen Flächen. - Acta Math. 50 (1927), $189-358$.

[10] Zieschang, H.: Über Automorphismen ebener diskontinuierlicher Gruppen, Math. Ann. 166 (1966), 148-167.

Research supported by NSF contract no. GP-9142. 